\title{
Silencing Hoxa2 reverses dexamethasone-induced dysfunction of MC3T3-E1 osteoblasts and osteoporosis in rats
}

\author{
Yuan Liu, ${ }^{1, A-D}$, Le Wang ${ }^{1, B, C}$, Youguo Yang ${ }^{1, B, C}$, Jianbin Xiong ${ }^{2, A, E, F}$ \\ ${ }^{1}$ Department of Rheumatology, Liuzhou People's Hospital, Guangxi Medical University, China \\ 2 Department of Orthopedics, Liuzhou People's Hospital, Guangxi Medical University, China \\ A - research concept and design; $B$ - collection and/or assembly of data; $C$ - data analysis and interpretation; \\ $D$ - writing the article; $E$ - critical revision of the article; $F$ - final approval of the article
}

\section{Address for correspondence}

Jianbin Xiong

E-mail: xjianbin56@163.com

\section{Funding sources}

Grant from the Key Laboratory Project

of Capital Medical University, Beijing, China

(No. 2015LCLB05).

Conflict of interest

None declared

Received on 0ctober 26, 2020

Reviewed on November 2, 2020

Accepted on February 19, 2021

Published online on May 13, 2021

\begin{abstract}
Background. Osteoporosis is damaging the health of women worldwide. Osteoporosis results from the imbalance between bone resorption and formation, which may be regulated by homeobox A2 (Hoxa2). However, the specific role and mechanism of Hoxa 2 in osteogenesis and dexamethasone (Dex)-induced osteoporosis remain unknown.
\end{abstract}

Objectives. The present study investigated the effect of Hoxaz on differentiation and osteoblastogenesis.

Materials and methods. Alkaline phosphatase staining and immunofluorescence staining were performed to evaluate the differentiation of MC3T3-E1 cells. Runt-related transcription factor 2 (RunX2), osteoprotegerin (OPG) and receptor activator of nuclear factor-kappa B ligand (RANKL) in Dex stimulated osteoblastic MC3T3-E1 cells, and Dex-induced osteoporotic rats were estimated using western blot and quantitative polymerase chain reaction (qPCR). Serum markers of bone turnover were determined using enzyme-linked immunosorbent assay (ELISA). Trabecular bones of femur tissues were observed using hematoxylin and eosin (H\&E) staining.

Results. Hoxa2 short hairpin RNA significantly promoted the differentiation of MC3T3-E1 cells and expression of Runx 2 and OPG in Dex-treated MC3T3-E1 cells and osteoporotic rats but inhibited the expression of RANKL. Furthermore, silencing Hoxa2 resulted in the upregulation of bone alkaline phosphatase but suppressed the expression of tartrate-resistant acid phosphatase and C-terminal cross-linked telopeptides of type I collagen.

Conclusions. Silencing Hoxa2 reversed the Dex-induced inhibition of osteoblastogenesis by modulating Runx2 and RANK-RANKL-OPG axis.

Key words: homeobox A2, osteoblast, dexamethasone, osteogenesis

Cite as

Liu Y, Wang L, Yang Y, Xiong J. Silencing Hoxa2 reverses dexamethasone-induced dysfunction of MC3T3-E1 osteoblasts and osteoporosis in rats. Adv Clin Exp Med. 2021;30(5):525-534. doi:10.17219/acem/133495

DOI

10.17219/acem/133495

\section{Copyright}

Copyright by Author(s)

This is an article distributed under the terms of the

Creative Commons Attribution 3.0 Unported (CC BY 3.0)

(https://creativecommons.org/licenses/by/3.0/) 


\section{Background}

The precise balance of bone turnover depends on the homeostasis of osteoblast-mediated bone formation and osteoclast-mediated bone resorption, which are mediated by a multitude of critical molecules working through various signaling pathways. ${ }^{1}$ Osteoporosis occurs due to the imbalance of bone formation and resorption, with excess resorption leading to reduced bone mass and increased bone fragility. The proliferation and differentiation of osteoblasts from undifferentiated mesenchymal cells play important roles in bone formation. ${ }^{2}$ Glucocorticoid-induced osteoporosis is regarded as the most common form of secondary osteoporosis and involves a reduction in osteoblastogenesis ${ }^{3}$; thus, Dex-treated osteoblast and animal models have been widely accepted as in vitro and in vivo models of osteoporosis. ${ }^{4,5}$ Although osteoporosis treatments based on the inhibition of bone resorption and promotion of bone formation are widely used, prolonged administration of these agents, such as bisphosphonates or estrogen receptor modulator, can lead to several adverse effects such as mandibular necrosis, venous thrombosis, cardiac arrhythmia, and renal inadequacy. ${ }^{6}$ Thus, more effective therapeutic strategies with fewer adverse reactions are urgently needed. To date, there is still considerable need for illustrating the effects of critical regulatory factors of osteoblastogenesis and for therapeutic targets associated with osteoporosis.

Currently, several signaling pathways and factors involved in osteoporosis have been determined. ${ }^{7}$ For instance, $R u n x 2$, which can act as a transcriptional regulator of bone metabolism, participates in the differentiation and formation of osteoblasts as well as signal transduction of specific pathways, including the osteoprotegerin $(\mathrm{OPG}) /$ receptor activator of nuclear factor $\mathrm{\kappa} B$ ligand (RANKL) pathway. ${ }^{8,9}$ Notably, a combination treatment with RANKL and receptor activator of nuclear factor $\mathrm{kB}$ (RANK) promotes the differentiation and proliferation of osteoclasts, and OPG has been found to inhibit osteoclast activation by competitively combining with RANK. Thus, both OPG and RANK are key molecules in bone formation and reconstruction. ${ }^{10}$ Bone formation is largely dependent on the proliferation and differentiation of osteoblasts. ${ }^{11-13}$ The ALP production is regarded as an indicator of osteogenesis, to a certain extent, reflecting the degree of osteoblast differentiation. ${ }^{14}$ MC3T3-E1 cells display developmental, sequential gene expression similar to that of osteoblasts during bone formation in vivo, which are considered a well-established model for studying osteoblast proliferation and differentiation. ${ }^{15,16}$

Homeobox A2 (Hoxa2), a unique transcription factor of the homeobox family, regulates many growth and development processes. Knockout of Hoxa2 in mice has been found to promote expression of the cartilage and bone-specific gene Runx2, which regulates the expression of bonespecific markers. ${ }^{17}$ Previous research has shown that Hoxa2 inhibits osteoblast differentiation by negatively regulating the osteogenic transcription factor $R u n \times 2 .{ }^{18}$ Moreover, downregulation of Hoxa2 via miR-135 facilitates the differentiation of adipose-derived stem cells (ADSCs) into osteoblasts via the Runx2 pathway. ${ }^{19}$ Osteoblast formation per se upregulates the Hoxa gene cluster expression, particularly of mid-cluster genes, and downregulates the expression of Hoxa7 and Hoxa10, differences of which in expression appear related to promoter methylation. Hoxa expression is profoundly regulated during osteoblast differentiation through canonical methylation-dependent mechanisms. ${ }^{20}$ However, the molecular mechanisms by which Hoxa2 influences differentiation and regeneration of osteoblasts, especially in glucocorticoid-induced osteoporosis, require further investigation to clarify whether Hoxa2 is essential for these processes and whether it is a suitable target for a gene therapy-based approach to osteoporosis.

\section{Objectives}

The aim of this study was to identify the precise role of Hoxa2 in glucocorticoid-induced suppression of osteogenesis. Dex-induced MC3T3-E1 osteoblasts and osteoporotic rats were respectively transfected and administrated with adenovirus-packaged Hoxa2 short hairpin RNA (shRNA) in vitro and in vivo to observe the effect of Hoxa2 on the differentiation of osteoblasts, as well as osteoporosis in rats.

\section{Materials and methods}

\section{Construction of recombinant adenovirus- delivered Hoxa2 shRNA}

Two shRNA sequences targeting Hoxa2 (Hoxa2 shRNA\#1: 5'-CCCACTGTTCCTAACTGCTTGTCAA-3' and Hoxa2 shRNA\#2: 5'-GCTCCCTGGACAGTCCTGTAGATA-3') were designed according to GenBank accession number (SEQ ID: nM_010451.2). The scrambled shRNA (5'-GCTGCTGGATTTGACCGAGAGACAA-3') was used as a negative control (Sangon Biotechnology, Shanghai, China). These shRNAs were packaged with adenovirus vector pHBAd-U6-GFP. The fluorescent intensity of cells was analyzed using a fluorescence microscope (Carl Zeiss, Jena, Germany) or a flow cytometer (FACS Calibur; Becton Dickinson, Franklin Lakes, USA).

\section{Cell culture and transfection}

The mouse osteoblastic cell line MC3T3-E1 was purchased from American Type Cell Culture (ATCC, Manassas, USA) and maintained in $\alpha$-Minimal Essential Medium (Gibco BRL, Rockville, USA) supplemented with $10 \% \mathrm{fe}-$ tal bovine serum (FBS), $100 \mathrm{U} / \mathrm{mL}$ of penicillin (Hyclone, Logan, USA) and $100 \mathrm{U} / \mathrm{mL}$ of streptomycin at $37^{\circ} \mathrm{C}$ in a $5 \% \mathrm{CO}_{2}$ atmosphere. The cells were passaged upon 
reaching $70 \%$ fusion using $0.25 \%$ trypsin, and cells of passages 2 through 4 were used for experiments.

MC3T3-E1 cells were divided into 5 groups and seeded at a density of $2 \times 10^{5}$ cells/well in six-well plates. The Dex group was pretreated with $100 \mathrm{nM}$ Dex for 72 h (Sigma-Aldrich, St. Louis, USA). MC3T3-E1 cells in the blank group were cultured with normal saline, and the Dex+scrambled shRNA group was treated with $100 \mathrm{nM}$ Dex for $24 \mathrm{~h}$ followed by transfection with scrambled shRNA for $48 \mathrm{~h}$. The Dex+Hoxa 2 shRNA\#1 group and Dex+Hoxa2 shRNA\#2 groups were treated with $100 \mathrm{nM}$ Dex for $24 \mathrm{~h}$ followed by transfection with Hoxa 2 shRNA\#1 or Hoxa 2 shRNA\#2 for $48 \mathrm{~h}$, respectively.

\section{Cell viability assay}

The commercially available Cell Counting Kit-8 (CCK-8) assay (Sigma-Aldrich) was used to assess cell viability according to the manufacturer's instructions. Cells in the logarithmic growth phase were seeded in 96-well culture plates at $3 \times 10^{3}$ cells/well. Then, $10 \mu \mathrm{L}$ of CCK-8 solution was added to each well for $2 \mathrm{~h}$ at $5 \mathrm{~h}, 24 \mathrm{~h}, 48 \mathrm{~h}$, and $72 \mathrm{~h}$ after cultivation. The absorbance at $450 \mathrm{~nm}$ in each well was measured using a microplate reader (BioTek, Winooski, USA), and experiments were performed in triplicate.

\section{ALP staining}

MC3T3-E1 cells were washed twice with phosphatebuffered saline (PBS) and fixed with $4 \%$ paraformaldehyde for $15 \mathrm{~min}$, followed by washing with $25 \mathrm{mM}$ Tris-Cl ( $\mathrm{pH}$ 9.0). Then, cells were stained with ALP dye solution containing $8 \mathrm{mM} \mathrm{MgCl} 2,0.4 \mathrm{mg} / \mathrm{mL}$ of $\alpha$-naphthyl phosphate, and $1 \mathrm{mg} / \mathrm{mL}$ of Fast Red TR (Sigma-Aldrich) for $30 \mathrm{~min}$ and rinsed with PBS to terminate the reaction. The ALP-positive cells (stained red) were counted under a fluorescence microscope (Carl Zeiss).

\section{Immunofluorescence staining}

MC3T3-E1 cells were seeded in Petri dishes and washed with PBS, and then cells were fixed in $4 \%$ formaldehyde for $30 \mathrm{~min}$ at room temperature. MC3T3-E1 cells were subsequently permeabilized with $0.5 \%$ Triton X-100 solution for $10 \mathrm{~min}$ and sealed with FBS for $30 \mathrm{~min}$. After that, the cells were incubated with the following primary antibodies: mouse anti-bone alkaline phosphatase (BALP) monoclonal antibody ( $1: 100$; Abcam, Cambridge, USA), and mouse anti-PICP monoclonal antibody (1:50; Abcam) at $4{ }^{\circ} \mathrm{C}$ overnight. After washed with PBS, the secondary goat-anti-mouse IgG-fluorescein isothiocyanate (FITC; 1:500; Abcam) was added to incubate cells for $1 \mathrm{~h}$, and nuclei were counterstained with DAPI (4', 6-diamidino2-phenylindole dihydrochloride). Finally, fluorescence staining was observed using a laser confocal fluorescence microscope (Carl Zeiss).

\section{Animal administration}

A total of 30 male Wistar rats (body weight $250-300 \mathrm{~g}$, 12 weeks old) were maintained under a standard pathogen-free environment at $22-25^{\circ} \mathrm{C}$ and $45-55 \%$ humidity with a $12 \mathrm{~h} \mathrm{light/dark} \mathrm{cycle.} \mathrm{The} \mathrm{rats} \mathrm{had} \mathrm{free} \mathrm{access}$ to water and a standard rodent diet. After acclimatization for 1 week, rats were randomly divided into 5 groups: control group (administered with normal saline); Dex group (daily subcutaneous injection with $0.1 \mathrm{mg} / \mathrm{kg}$ Dex for 6 weeks); Dex+scramble shRNA group; Dex+Hoxa 2 shRNA\#1 group; and Dex+Hoxa 2 shRNA\# 2 group (6 weeks of subcutaneous injection of $0.1 \mathrm{mg} / \mathrm{kg}$ of Dex plus an intravenous injection of $1 \times 10^{9} \mathrm{pfu} / \mathrm{mL}$ of scramble shRNA or Hoxa 2 shRNA\#1 or Hoxa 2 shRNA\#2 once every 2 days for 4 weeks). The serum and right femurs were collected from the sacrificed animals at the endpoint. The animal experiment was performed in accordance with the protocol approved by the Ethics Committee of Liuzhou People's Hospital 2020 (China; approval No. KY-E-14-d).

\section{RT-qPCR}

Gene expression in MC3T3-E1 cells and proximal femoral tissues was assessed using quantitative polymerase chain reaction (qPCR). Total RNA extraction was performed using TRIzol ${ }^{\circledR}$ reagent (Invitrogen, Carlsbad, USA) according to the manufacturer's instructions. The RNA concentration and purity were verified using the Nanodrop 2000 (Thermo Fisher Scientific, Rockford, USA). For reverse transcription, cDNA was synthesized chemically with $2 \mu \mathrm{g}$ of total RNA using the RevertAid First Strand cDNA Synthesis Kit (MBI Fermentas, Vilnius, Lithuania). The qPCR was performed with the SYBR Green PCR kit (Qiagen, Crawley, UK) using an ABI quantitative PCR 7300 system (Applied Biosystems, Foster City, USA). The amplification conditions for PCR were $95^{\circ} \mathrm{C}$ for $5 \mathrm{~min}$ followed by 40 cycles of $10 \mathrm{~s}$ of denaturation at $95^{\circ} \mathrm{C}, 20 \mathrm{~s}$ of annealing at $60^{\circ} \mathrm{C}$ and $30 \mathrm{~s}$ of extension at $72^{\circ} \mathrm{C}$. The following primers were used: Hoxa2, forward: 5'-CTGTGGAGCTGGCCTAAACA-3' and reverse: 5'-GCAAAGCCACCTGGTCAAAG-3'; Runx2, forward: 5'-GACTGTGGTTACCGTCATGGC-3' and reverse:5'-ACTTGGTTTTTCATAACAGCGGA-3'; OPG, forward: 5'-AAAGCACCCTGTAGAAAACA-3' and reverse: 5'-CCGTTTTATCCTCTCTA CACTC-3'; RANKL, forward: 5'-TATGATGGAAGGCTCATGGT-3' and reverse: 5'-TGTCCTGAACTTTGAAAGCC-3'; GAPDH, forward: 5'-TGGCCTTCCGTGTTCCTAC-3' and reverse: 5'-GAGTTGCTGTTGAAGTCGCA-3'. Upon completion of the reaction, the amplification curve and melting curve were assessed. The relative expression of each mRNA was compared to that of GAPDH and calculated using the $2^{-\Delta \Delta \mathrm{Ct}}$ method. 


\section{Western blot}

Protein was extracted using lysis buffer containing $1 \mathrm{mM}$ phenylmethane sulfonyl fluoride (PMSF; Applygen, Beijing, China), and the total protein concentration was measured using a BCA assay kit (Sigma-Aldrich). Protein samples were separated with $10 \%$ sodium dodecyl sulfate polyacrylamide gel electrophoresis (SDS-PAGE) and transferred to nitrocellulose membranes (Applygen). Non-specific binding was blocked by adding $5 \%$ fat-free milk in Tris-buffered saline containing 0.1\% Tween-20 (TBST). Membranes were then incubated at $4^{\circ} \mathrm{C}$ overnight with primary antibodies against GAPDH (1:500; Santa Cruz Biotechnology, Santa Cruz, USA), rabbit anti-Hoxa 2 polyclonal antibodies ( $1: 300 ; \mathrm{Abcam})$, mouse anti-RANKL monoclonal antibody ( $1: 500$; Abcam), rabbit anti-Runx 2 monoclonal antibody $(1: 500$; Cell Signaling Technology, Danvers, USA), and rabbit anti-OPG polyclonal antibody ( $1: 800 ; \mathrm{Abcam})$. The membranes were washed with TBST and then incubated with goat anti-mouse/rabbit secondary antibodies (1:1000; Santa Cruz Biotechnology) at room temperature for $1 \mathrm{~h}$. Protein bands were visualized using enhanced chemiluminescence (ECL) reagent (Thermo Fisher Scientific), and GAPDH was used as an endogenous protein for normalization.

\section{Histopathological analysis}

The femur was fixed with $4 \%$ neutral buffered formalin for 7 days at $4{ }^{\circ} \mathrm{C}$ and decalcified in $5 \%$ ethylenediaminetetraacetic acid (EDTA) for 3 weeks. The tissues then were embedded in paraffin wax and cut into $5 \mu \mathrm{m}$ sections, which were stained with hematoxylin and eosin (H\&E). The histopathological changes of bone cortex and trabecula were observed under a light microscope (model IX53; Olympus Corp., Tokyo, Japan).

\section{ELISA}

Serum biomarkers for bone turnover, including BALP, tartrate-resistant acid phosphatase 5b (TRAP5b) and carboxy-terminal of type I collagen cross-links (CTX-I), were assessed using enzyme-linked immunosorbent assay (ELISA) kits (Immunodiagnostic Systems, Fountain Hills, USA) according to the manufacturer's instructions.

\section{Statistical analysis}

Data are presented as mean \pm standard error of the mean (SEM). One-way analysis of variance (ANOVA) was used to assess the differences in variables among multiple groups, and comparisons within groups were made using post hoc tests. In addition, data obtained from multiple measurements of the same dependent variable at different time points were compared using repeated-measures ANOVA. The statistical significance was defined by a value of $\mathrm{p}<0.05$. All statistical comparisons were performed using SPSS v. 17.0 (SPSS Inc., Chicago, USA).

\section{Results}

\section{The upregulated Hoxa2 induced by Dex in MC3T3-E1 cells was overturned by Hoxa2 shRNA}

The sequences of Hoxa2 shRNA and scrambled shRNA are presented in Fig. 1A. MC3T3-E1 cells were transfected with scrambled shRNA, Hoxa2 shRNA\#1 and Hoxa2 shRNA\#2, respectively. Green fluorescence was observed post-transfection, indicating that shRNAs were transfected into cells successfully (Fig. 1B,C). The silencing effects of Hoxa2 shRNAs were evaluated using RT-PCR and western blot analysis. Results showed that Dex promoted the level of Hoxa2 at both mRNA and protein levels. However, the mRNA expression of Hoxa2 in MC3T3-E1 cells transfected with Hoxa2 shRNA\#1 or Hoxa2 shRNA\#2 was significantly decreased, consistent with the downregulation of Hoxa 2 protein level. The scrambled shRNA did not affect Hoxa2 expression (Fig. 1D,E). These findings demonstrated that the level of Hoxa2 was increased in Dex-induced MC3T3-E1 cells, and the Hoxa2 shRNAs were effective.

\section{Knockdown of Hoxa2 attenuated the inhibitory effect of Dex on osteoblast differentiation in MC3T3-E1 cells}

The cell viability was determined using the CCK-8 assay. The viability of MC3T3-E1 cells exposed to Dex was drastically weakened compared to the blank group at $24 \mathrm{~h}$, $48 \mathrm{~h}$ and $72 \mathrm{~h}$, whereas Hoxa 2 shRNA\#1 and Hoxa2 shRNA\#2 elevated the cell viability, hinting that the important role of Hoxa2 in MC3T3-E1 cell viability (Fig. 2A). BALP and PICP, which reflect activity of the osteoblasts, ${ }^{21,22}$ were selected to determine the differentiation and function of osteoblasts. The enrichment of BALP and PICP expressions was observed in untreated MC3T3-E1 cells, and their levels were greatly reduced following treatment with Dex. Moreover, Hoxa2 shRNAs rescued the expressions of BALP and PICP partly compared with the Dex+scrambled shRNA group (Fig. 2B,C). Collectively, these results suggested that Hoxa 2 affected the viability of MC3T3-E1 cells, and the inhibitory effect of Dex on osteoblast differentiation was reversed by silencing Hoxa2.

\section{Hoxa2 knockdown alleviated the inhibitory effect of Dex on osteogenesis in MC3T3-E1 cells}

The ALP is regarded as an indicator of osteogenesis, to a certain extent, reflecting the degree of osteoblast differentiation. ${ }^{23}$ Results showed that the number of positive 
A

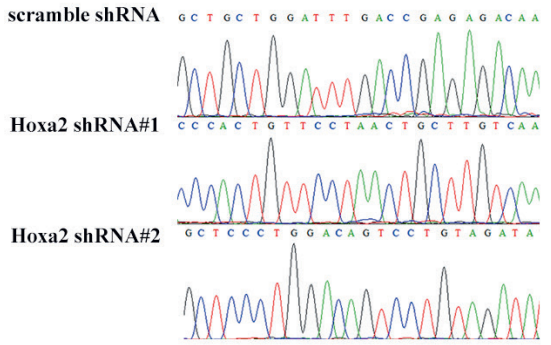

D

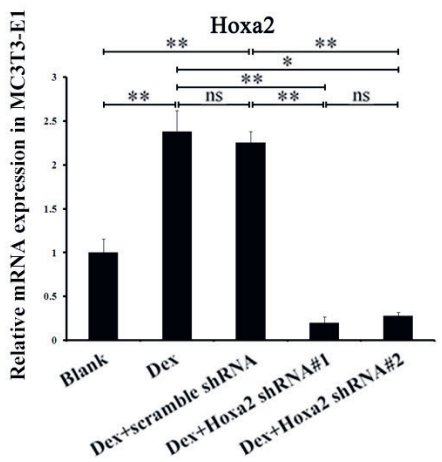

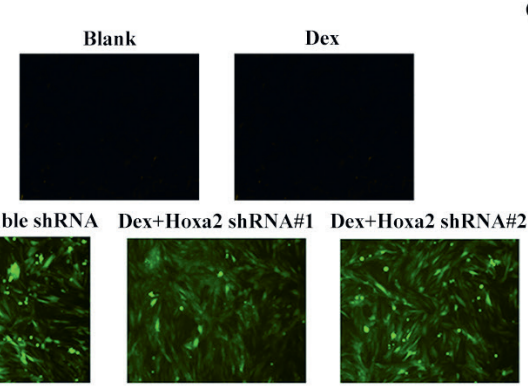

C

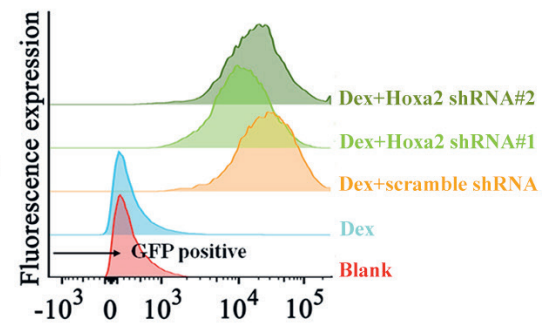

E
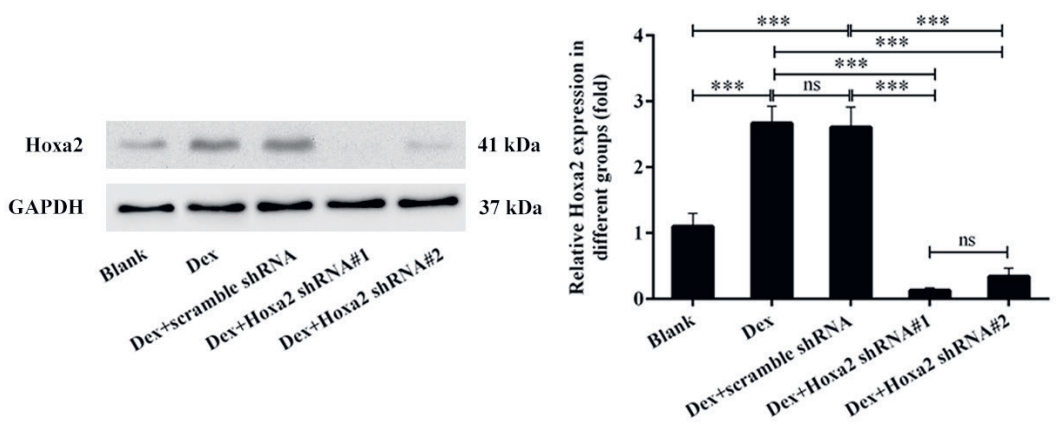

Fig. 1. The upregulated Hoxa2 induced by dexamethasone (Dex) in MC3T3-E1 cells was overturned by Hoxa2 shRNA. A. Gene sequencing was used to identify recombinant adenoviruses for Hoxa2 shRNA1 and shRNA2; B. Fluorescence microscopy was used to examine the expression of green fluorescence in MC3T3-E1 cells after transfection with the control shRNA and 2 Hoxa2 shRNAs. Scale bar: 50 um; C. The transfection efficiency of Hoxa2 shRNA in MC3T3-E1 cells was determined with flow cytometry after the treatment of cells with Dex; D and E. Silence effect of Hoxa2 shRNA in MC3T3-E1 cells was confirmed with real-time PCR (RT-PCR) and western blot

${ }^{*} \mathrm{p}<0.05 ;{ }^{* *} \mathrm{p}<0.01 ;{ }^{* * *} \mathrm{p}<0.001 ;$ ns - not significant.

ALP-positive cells (red staining) was reduced after Dex stimulation. In contrast, numerous ALP-positive cells were observed following transfection of Hoxa2 shRNA\#1 or Hoxa 2 shRNA\#2 (Fig. 3A). To further confirm the role of Hoxa2 in Dex-induced osteogenesis dysfunction, we analyzed the expression levels of several transcriptional factors and signaling molecules known to be involved in the regulation of osteogenesis, including Runx2, OPG, and RANKL. ${ }^{23-25}$ Dex treatment decreased the mRNA expressions of osteoblastogenic molecules Runx2 and OPG in MC3T3-E1 cells compared with the blank group (Fig. 3B,C). In contrast, the mRNA expression of the negative osteogenesis regulator RANKL was significantly higher in the Dex group than in the blank group (Fig. 3D). Similar results were observed in the protein expression of these molecules (Fig. 3E). Furthermore, Hoxa2 shRNA resulted in an increase of Runx2 and OPG at both mRNA and protein expression, but also in a decrease of RANKL expression compared with the Dex+scrambled shRNA group (Fig. 3B-E). The above findings suggested that Hoxa 2 shRNA counteracted the inhibitory effects of Dex on osteogenesis.

\section{Hoxa2 shRNA inhibited Dex-induced bone loss}

Adenovirus-packaged Hoxa2 shRNA\#1 was selected for in vivo experiments. As shown in Fig. 4A, strong green fluorescence in femur tissues was observed after administration of scramble shRNA and Hoxa 2 shRNA\#1, suggesting that the adenovirus-packaged shRNAs were successfully injected into rats. The mRNA level of Hoxa2 in rat right femurs was significantly increased following Dex treatment, while Hoxa 2 shRNA\#1 markedly inhibited the expression of Hoxa2 (Fig. 4B). Given the potential role of Hoxa2 in Dex-induced osteoporosis, we further evaluated the histological changes of femur tissues in response to Hoxa2 knockdown. As shown in Fig. 4D, trabecular bones of femur tissues appeared rupture and reduction in Dex-induced osteoporotic rats compared to the control group, indicating the validity of the osteoporotic rat model. However, Hoxa 2 shRNA administration increased bone mass and improved structure to a comparable level with the normal group.

\section{Hoxa2 was required for the osteogenesis and bone resorption regulated by Dex}

In addition, the mRNA levels of Runx2 and OPG in femur tissues were both reduced following Dex treatment. However, Hoxa2 shRNA\#1 reversed the downregulation of Runx2 and OPG induced by Dex (Fig. 4A,B). Dex promoted the expression of RANKL in femur tissues, while it was significantly decreased after administration of Hoxa2 shRNA\#1 (Fig. 4C). The protein levels of Runx2, OPG and RANKL in each group were consistent with mRNA 

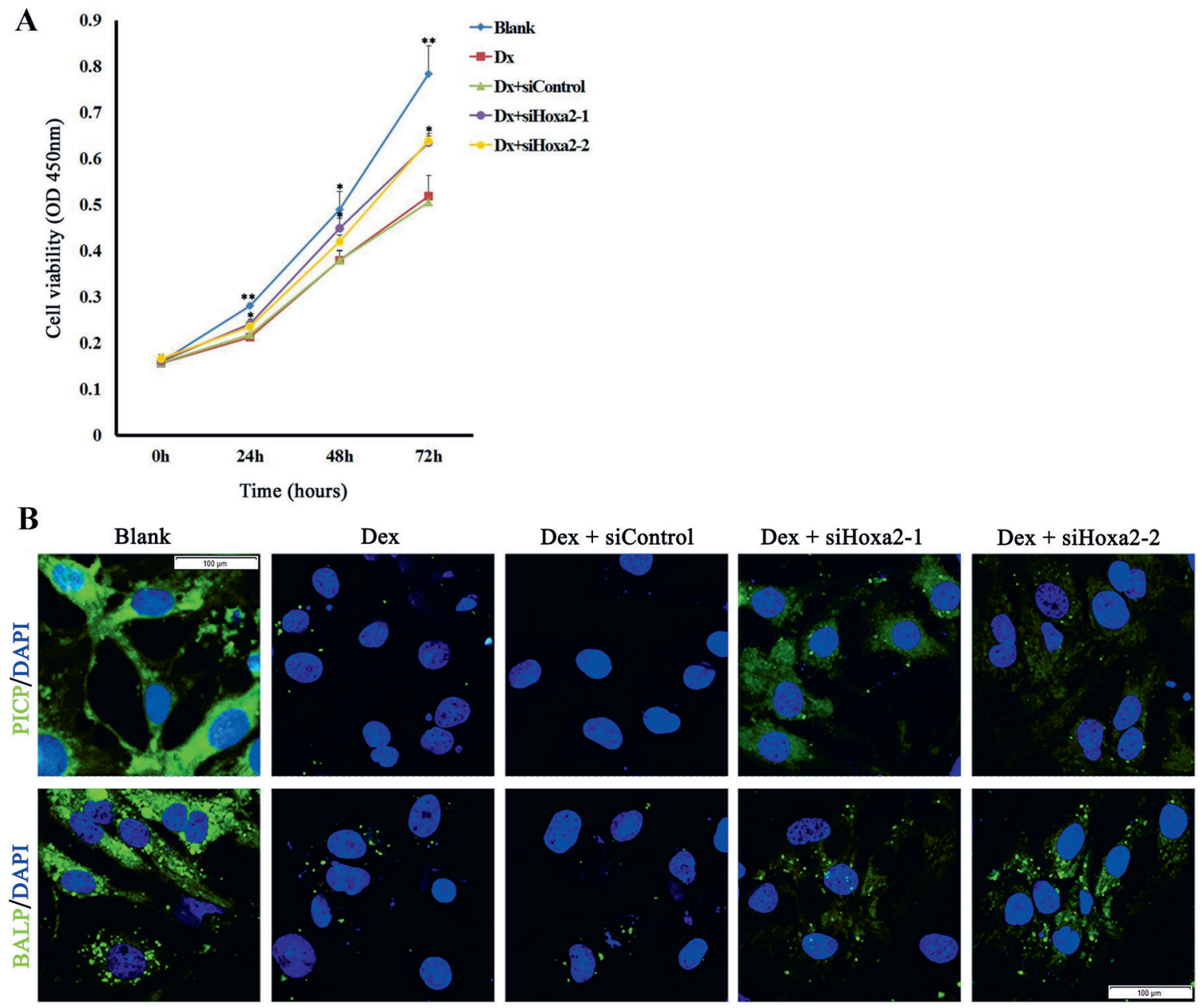

$\mathrm{C}$
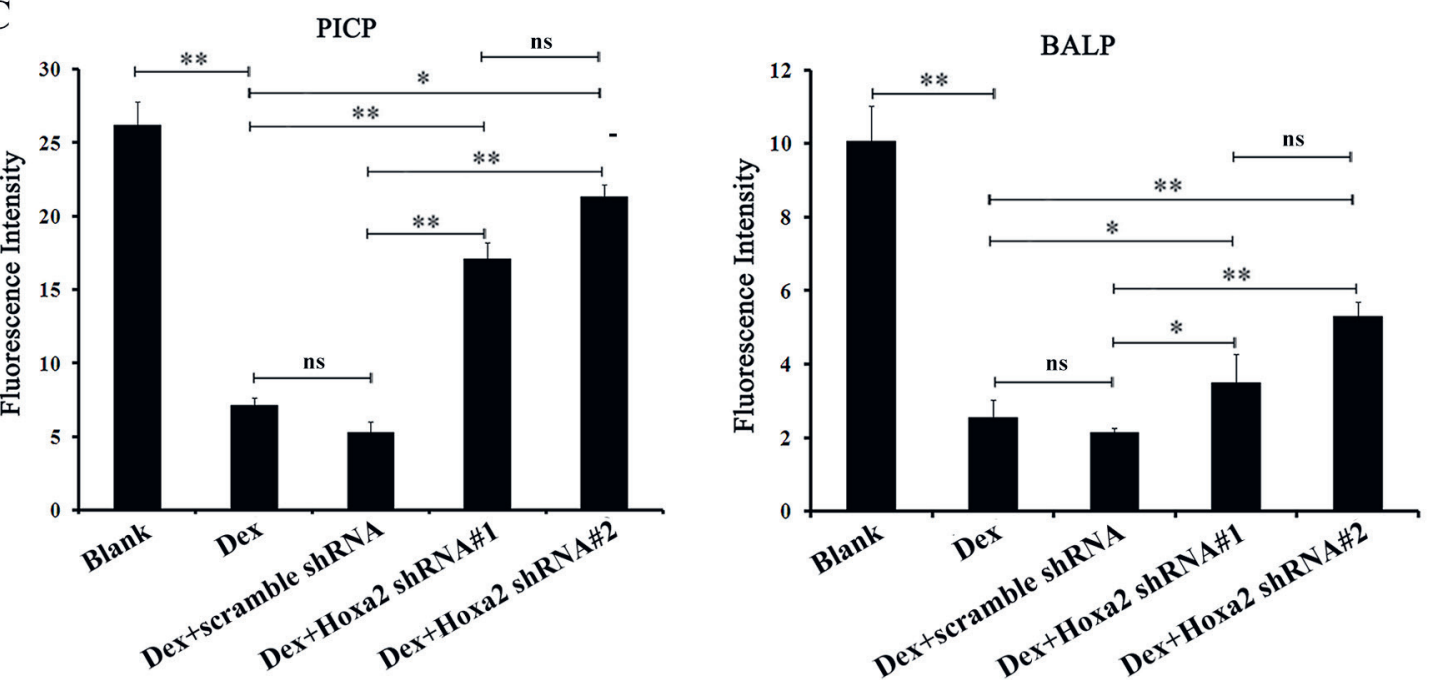

Fig. 2. Knockdown of Hoxa2 attenuated the inhibitory effect of Dex on osteoblast differentiation in MC3T3-E1 cells. A. MC3T3-E1 cells viability after stimulation with Dex and Hoxa2 shRNA; B and C. The expressions BALP and PICP in osteoblasts were determined with immunofluorescence staining. DAPI (blue) was used as a nuclear stain, and the expression of bone turnover markers is represented by green staining. Scale bar: $10 \mu \mathrm{m}$. Immunofluorescence staining of BALP and PICP was quantified using Image Pro Plus 4.5 software (Media Cybernetics, Silver Spring, USA)

levels (Fig. 4D). These results indicated that silencing Hoxa2 rescued the expressions of osteogenetic molecules in vivo. Compared with the control group, serum markers of bone resorption, including TRAP5b and C-terminal cross-linked telopeptides of type I collagen (CTX-1), were significantly increased in the Dex groups. However, Dex reduced the levels of bone formation marker BALP in comparison with the control group (Fig. 4E-G). Consistent with the beneficial effects on histological changes, Hoxa2 shRNA administration resulted in a reduction of serum 


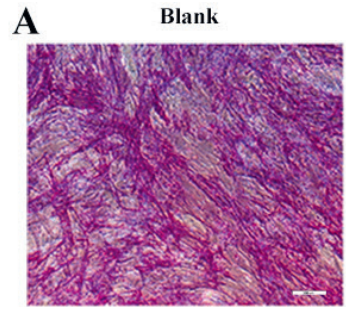

$\mathbf{B}$

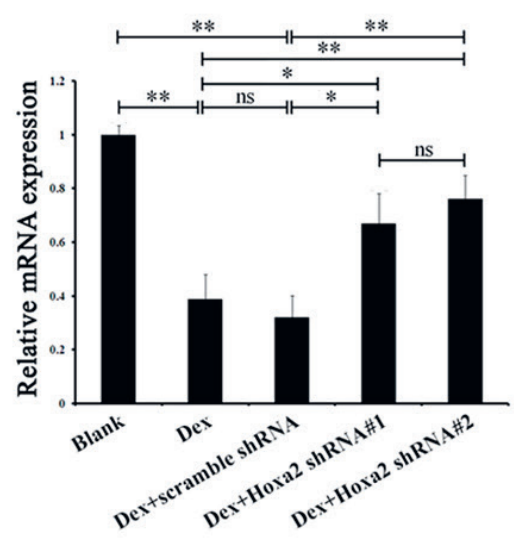

E

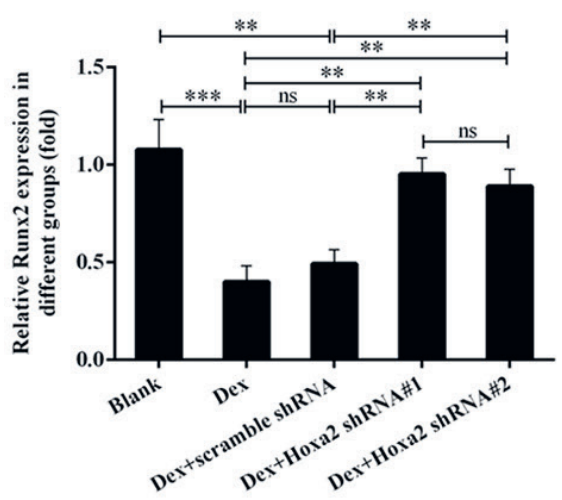

Dex

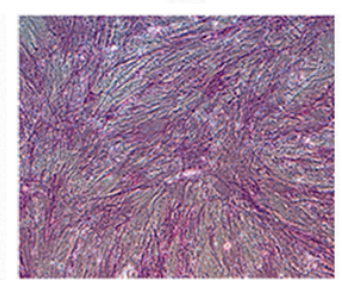

C

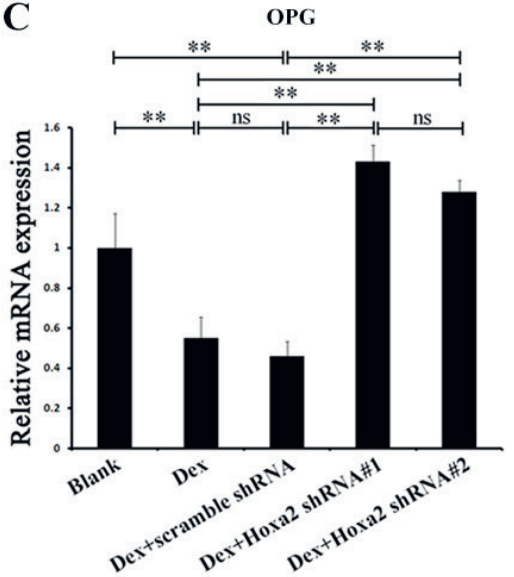

Dex+Hoxa2 shRNA\#1

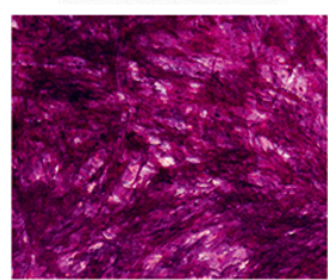

D
Dex+Hoxa2 shRNA\#2

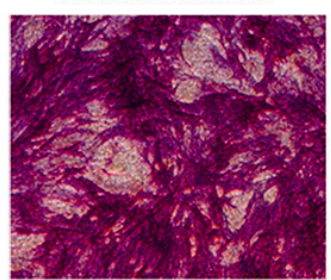

RANKL

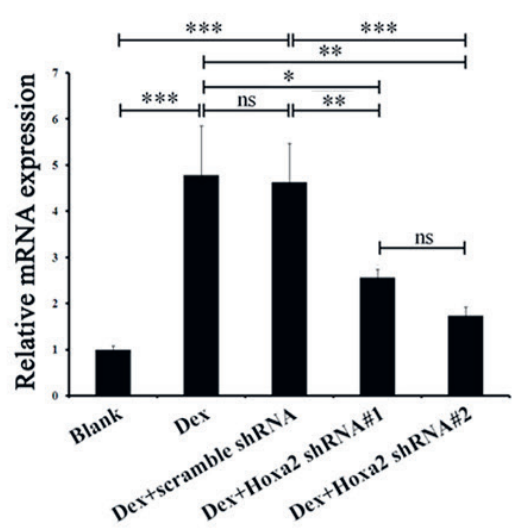

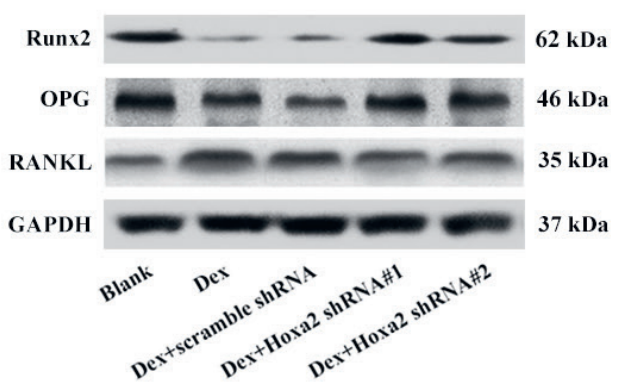
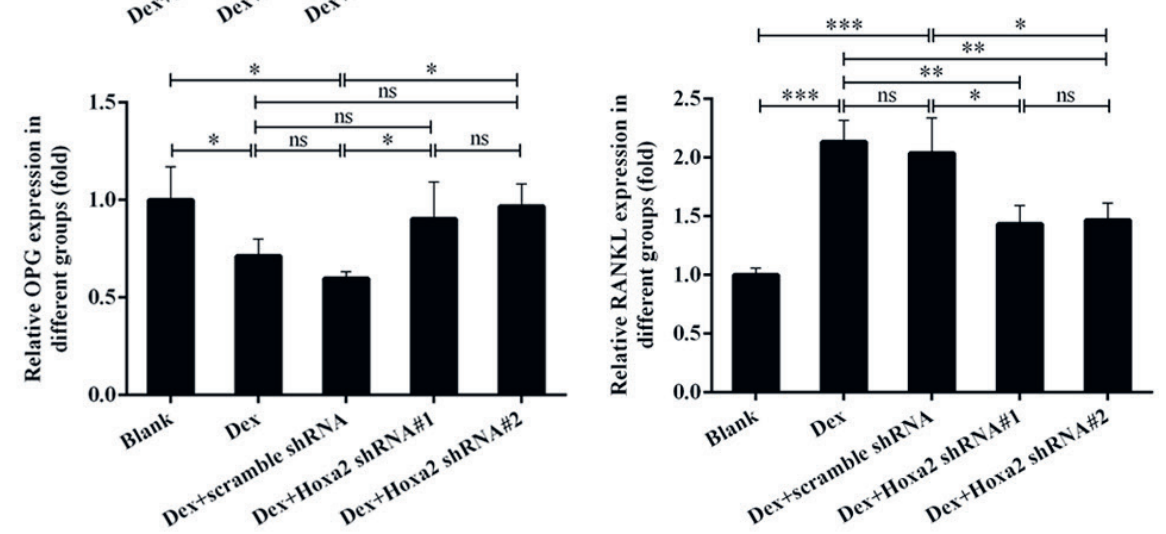

Fig. 3. Hoxa2 knockdown alleviated the inhibitory effect of Dex on osteogenesis in MC3T3-E1 cells. A. Differentiation of MC3T3-E1 cells was evaluated with ALP staining. The degree of red staining represented the number of ALP-positive cells and the extent of differentiation. Scale bar: $100 \mu$ m; B-D. RT-qPCR results show the effect of Hoxa2 shRNA on mRNA levels of Runx2, OPG and RANKL; E. Western blot showing the effect of Hoxa2 shRNA on protein levels of Runx2, OPG and RANKL

${ }^{*} p<0.05 ;{ }^{* *} p<0.01 ; * * p<0.001 ;$ ns - not significant.

TRAP5b and CTX-1, and an increase of BALP expression compared to the Dex group (Fig. 4E-G). The comprehensive results suggested that silencing Hoxa2 could reverse the Dex-mediated bone resorption and trabecular bone reduction.

\section{Discussion}

The normal process of bone metabolism is maintained by collaboration between osteoblasts and osteoclasts. Proliferation and differentiation of osteoblasts are responsible for 
A

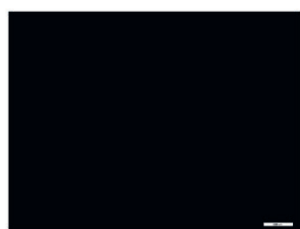

Dex+scramble shRNA
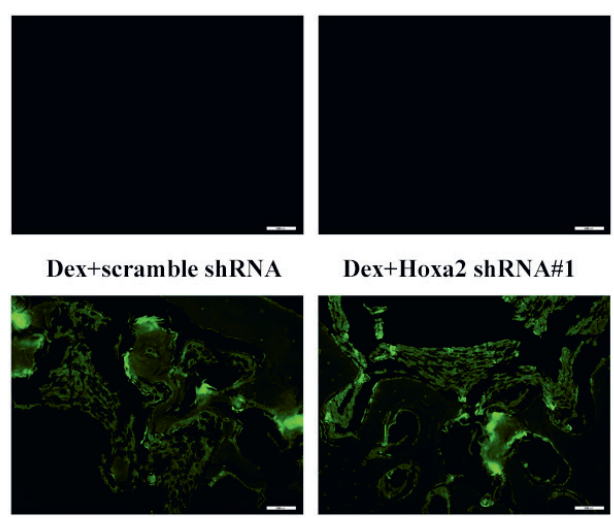

Dex+Hoxa2 shRNA\#1

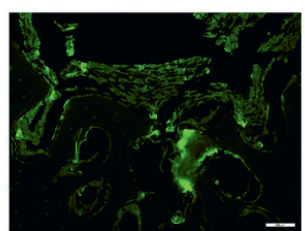

B

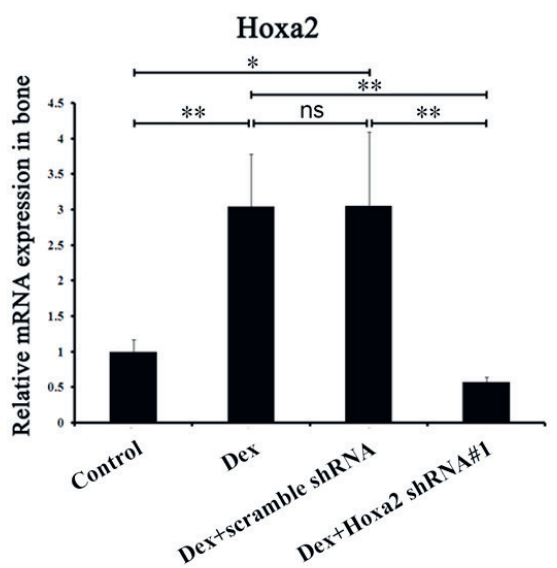

Fig. 4. Hoxa2 shRNA inhibited Dex-induced bone loss. A. Tissue targeting of Hoxa2 shRNA\#1 in rats. Scale bar: $100 \mu \mathrm{m}$; B. Silence effect of Hoxa2 shRNA\#1 in bone tissue was confirmed with RT-qPCR C. Histopathological changes of femur tissue in rats from a different group at the endpoint of the experiment; scale bar $100 \mu \mathrm{m}$

${ }^{*} \mathrm{p}<0.05$; ${ }^{* *} \mathrm{p}<0.01$; ns - not significant.
C

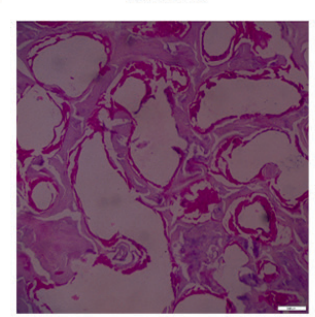

Dex

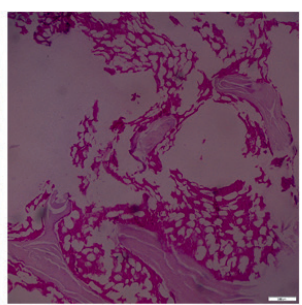

Dex+scramble shRNA

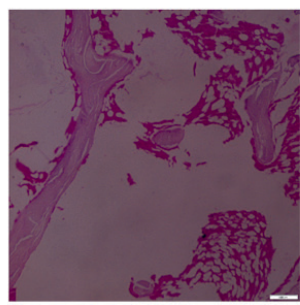

Dex+Hoxa2 shRNA\#1

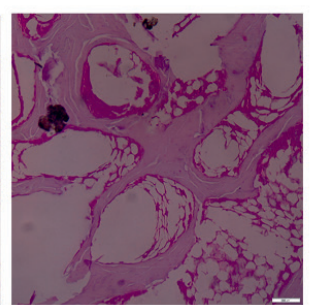

bone formation. ${ }^{26,27}$ To the best of our knowledge, MC3T3-E1 cells are a commonly used model for studying the function of osteoblasts. ${ }^{15,16}$ Hoxa2 is one of the Hox homeodomain family transcription factors and has been found to regulate bone metabolism and skeletal morphogenesis. ${ }^{17}$ Gersch et al. demonstrated that Hoxa-2 was upregulated in active osteoblasts during bone regeneration, and Dobreva et al. also reported higher expression of Hoxa2 in SATB2 ${ }^{-/}$osteoblasts. ${ }^{28,29}$ In view of the previous study, in which Hoxa 2 was shown to play a separate role in antagonizing bone formation, ${ }^{30}$ we sought to explore the potential effect of Hoxa2 on Dex-induced osteoporosis. Osteoporosis animal models were successfully developed in Dex-induced rats, with disease manifesting itself in pathological changes of the femur. ${ }^{31}$ Consistent with previous findings, we confirmed that Hoxa 2 was overexpressed in mouse osteoblast-like MC3T3-E1 cells and bone tissue of rats. Besides, Dex promoted Hoxa $2 \mathrm{mRNA}$ and protein expression. The above results promoted the hypothesis that Hoxa2 could be involved in the Dex-induced osteoblasts dysfunction and osteoporosis.

Another study has provided evidence that osteoblasts exposed to Dex display significant reductions in Col-I expression and ALP activity. ${ }^{32}$ Dex suppressed the osteogenic differentiation of bone marrow-derived mesenchymal stem cells and let-7f-5p expression, which could be reversed by the downregulation of TGFBR $1 .{ }^{33}$ This data was consistent with our findings showing that the expression of BALP and PICP was decreased in Dex-treated MC3T3-E1 cells. Notably, the expression of BALP and PICP was restored partially after Hoxa 2 knockdown. The homeostasis between osteoblasts and osteoclasts is mediated by various signaling molecules, including Run $\times 2^{34,35}$ and the RANKRANKL-OPG axis, imbalance of which leads to bone metabolism dysfunction. ${ }^{1}$ Franceschetti et al. found that RANKL effects on bone differentiation required PI3 kinase using computational analyses of several pathways in RANKL-driven differentiation of murine bone marrow osteoclast precursors. ${ }^{36} \mathrm{PI} 3 \mathrm{~K}$ was shown to mediate BMP2 induction of osteogenic differentiation. ${ }^{37}$ PI3K is involved in osteoblast adhesion to extracellular matrix and titanium..$^{38}$ In our study, silencing Hoxa 2 in Dex-induced osteoblasts and osteoporotic rats resulted in the upregulation of Runx2 and OPG as well as downregulation of RANKL. In line with its promotional effect on osteoblasts differentiation, Hoxa 2 regulating osteogenesis may be related to the Runx2 and RANK-RANKL-OPG axis. Thus, these findings revealed the underlying mechanism of Hoxa2 in osteoporosis. In addition, RANKL effects on bone differentiation were regulated by PI3K, and Hoxa2 affected the osteoblasts differentiation by downregulating the RANKL. Whether Hoxa 2 requires modulation of PI3 kinase activity warrants further investigation.

In Dex-induced osteoporotic rats, bone turnover biomarkers BALP, TRAP5b and CTX-1 have been identified to predict the activity and function of osteoblasts during the metabolic process. ${ }^{39}$ However, bone turnover biomarker expression varies throughout different metabolic statuses. ${ }^{12}$ The serum TRAP5b and CTX-1 were markedly reduced by the administration of Hoxa2 shRNA, while the BALP showed a significant increase compared to the Dex group, indicating that Hoxa2 shRNA inhibited osteoclasts activity and bone resorption. 

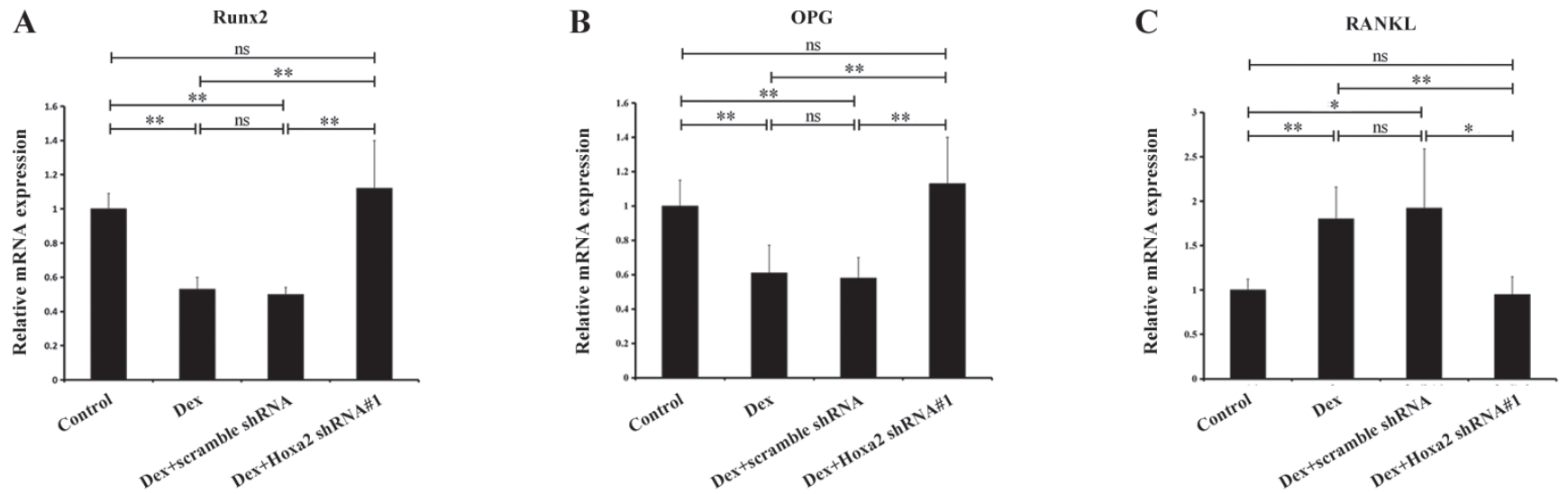

D
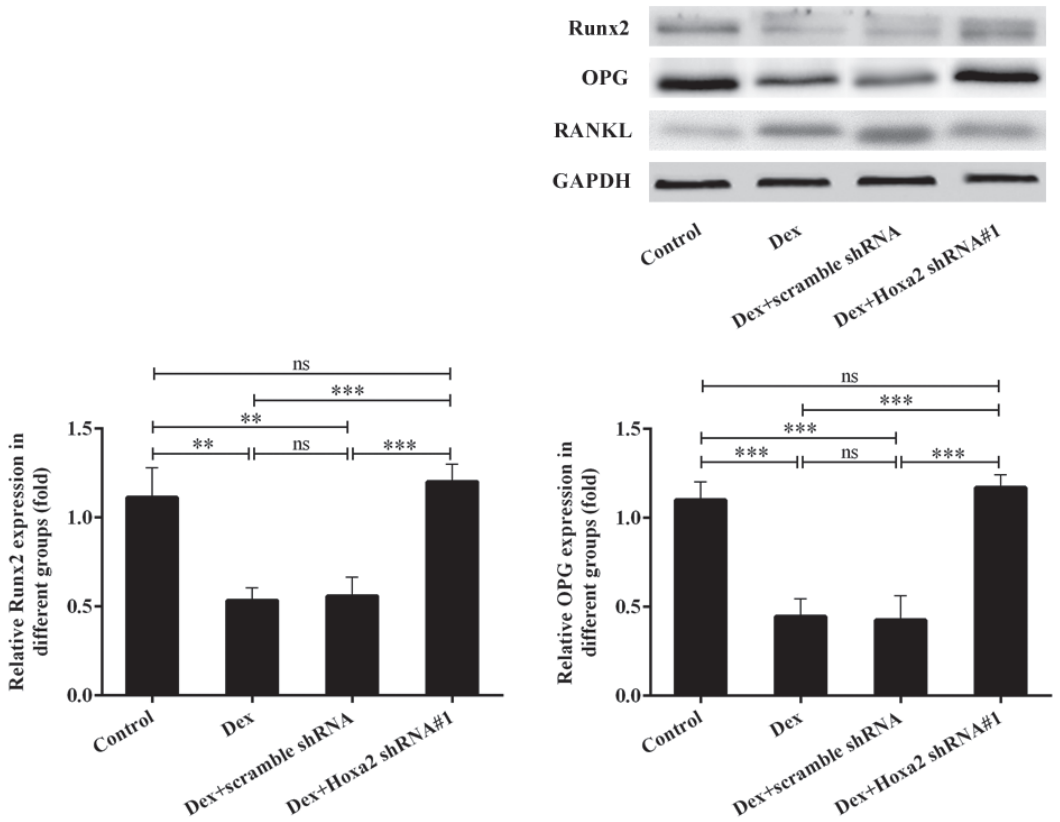

$62 \mathrm{kDa}$

$6 \mathrm{kDa}$

5 kDa

$37 \mathrm{kDa}$
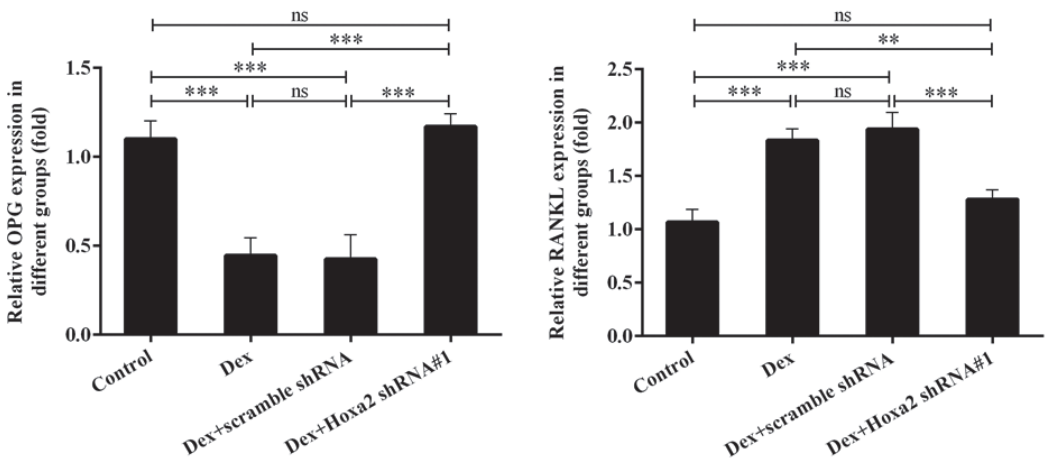

E
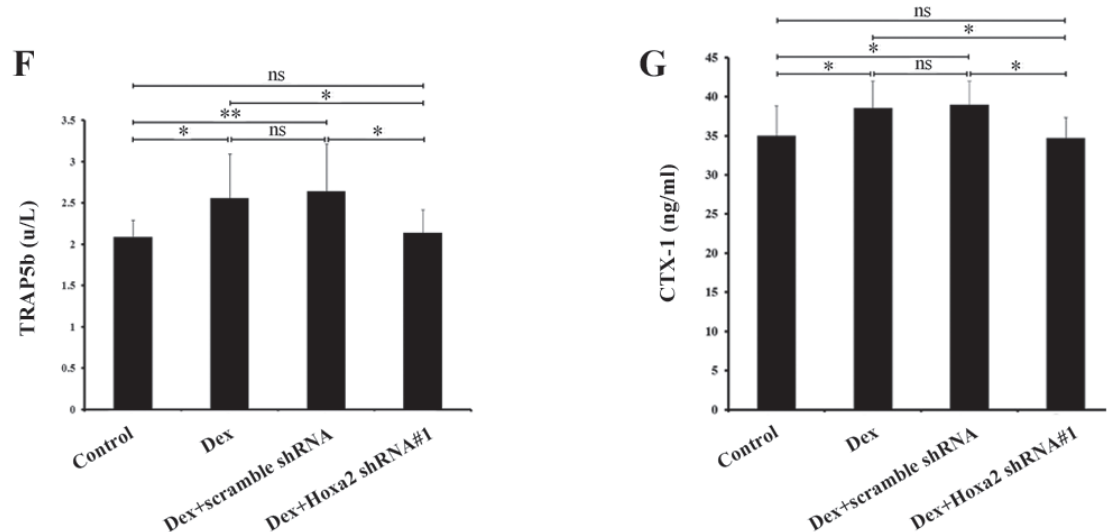

Fig. 5. Hoxa2 was required for the osteogenesis and bone resorption regulated by Dex. A-D. RT-qPCR and western blot showing the effect of Hoxa2 shRNA\#1 on mRNA and protein levels of Runx2, OPG and RANKL in Dex-induced osteoporotic rats; E-G. Bone turnover markers, including BALP, TRAP5b and CTX-1, in the serum of rats were evaluated using ELISA

${ }^{*} \mathrm{p}<0.05 ;{ }^{* *} \mathrm{p}<0.01 ;{ }^{* *} \mathrm{p}<0.001 ; \mathrm{ns}-$ not significant.

\section{Conclusions}

These findings provided evidence that Hoxa 2 was involved in the differentiation and osteogenesis of MC3T3E1 osteoblasts. Hoxa2 knockdown relieved Dex-regulated osteoporosis via modulating the Runx2 and the RANKRANKL-OPG axis. Our research provided an experimental basis for investigating a potential mechanism of bone metabolism in glucocorticoid-induced osteoporosis. Further studies are expected to reveal the complete regulatory 
mechanisms of Hoxa 2 in the pathogenesis of osteoporosis and its potential as a gene therapy target for the treatment of osteoporosis.

\section{ORCID iDs}

Yuan Liu (1) https://orcid.org/0000-0002-3913-0588

Le Wang (10 https://orcid.org/0000-0003-4426-9717

Youguo Yang (1) https://orcid.org/0000-0001-7215-8585

Jianbin Xiong (1) https://orcid.org/0000-0002-3946-0616

\section{References}

1. Huang W, Yang S, Shao J, Li YP. Signaling and transcriptional regulation in osteoblast commitment and differentiation. Front Biosci. 2007;12:3068-3092. PMID:17485283

2. Pittenger MF, Mackay AM, Beck SC, et al. Multilineage potential of adult human mesenchymal stem cells. Science. 1999;284(5411):143-147. doi:10.1126/science.284.5411.143

3. Adami G, Saag KG. Glucocorticoid-induced osteoporosis update. Curr Opin Rheumatol. 2019;31(4):388-393. doi:10.1097/BOR.00000000 00000608

4. Chen Z, Xue J, Shen T, Ba G, Yu D, Fu Q. Curcumin alleviates glucocorticoid-induced osteoporosis by protecting osteoblasts from apoptosis in vivo and in vitro. Clin Exp Pharmacol Physiol. 2016;43(2):268-276. doi:10.1111/1440-1681.12513

5. Shi C, Qi J, Huang P, et al. MicroRNA-17/20a inhibits glucocorticoidinduced osteoclast differentiation and function through targeting RANKL expression in osteoblast cells. Bone. 2014;68:67-75. doi:10. 1016/j.bone.2014.08.004

6. Yamauchi M, Sugimoto T. Status and issues of medical treatment for osteoporosis [in Japanese]. Nihon Rinsho. 2015;73(10):1621-1627. PMID:26529921

7. Iñiguez-Ariza NM, Clarke BL. Bone biology, signaling pathways, and therapeutic targets for osteoporosis. Maturitas. 2015;82(2):245-255. doi:10.1016/j.maturitas.2015.07.003

8. Chen D, Zhao M, Mundy GR. Bone morphogenetic proteins. Growth Factors. 2004;22(4):233-241. doi:10.1080/08977190412331279890

9. Gaur T, Lengner $\mathrm{CJ}$, Hovhannisyan $\mathrm{H}$, et al. Canonical WNT signaling promotes osteogenesis by directly stimulating Runx2 gene expression. J Biol Chem. 2005;280(39):33132-33140. doi:10.1074/jbc.M500608200

10. Khosla S. Minireview: The OPG/RANKL/RANK system. Endocrinology. 2001;142(12):5050-5055. doi:10.1210/endo.142.12.8536

11. Butler JS, Queally JM, Devitt BM, Murray DW, Doran PP, O'Byrne JM. Silencing $D k k 1$ expression rescues dexamethasone-induced suppression of primary human osteoblast differentiation. BMCMusculoskelet Disord. 2010;11:210. doi:10.1186/1471-2474-11-210

12. Camassa JA, Diogo CC, Bordelo JPA, et al. Tartrate-resistant acid phosphate as biomarker of bone turnover over the lifespan and different physiologic stages in sheep. BMC Vet Res. 2017;13(1):239. doi:10.1186/ s12917-017-1170-9

13. Ducy P, Zhang R, Geoffroy V, Ridall AL, Karsenty G. Osf2/Cbfa1: A transcriptional activator of osteoblast differentiation. Cell. 1997;89(5): 747-754. doi:10.1016/s0092-8674(00)80257-3

14. Taylor LM, Turksen K, Aubin JE, Heersche JN. Osteoclast differentiation in cocultures of a clonal chondrogenic cell line and mouse bone marrow cells. Endocrinology. 1993;133(5):2292-2300. doi:10.1210/endo. 133.5.7691585

15. Fu C, Xu D, Wang CY. Alpha-lipoic acid promotes osteoblastic formation in $\mathrm{H}_{2} \mathrm{O}_{2}$-treated MC3T3-E1 cells and prevents bone loss in ovariectomized rats. J Cell Physiol. 2015;230(9):2184-2201. doi:10.1002/ jсp. 24947

16. Quarles LD, Yohay DA, Lever LW, Caton R, Wenstrup RJ. Distinct proliferative and differentiated stages of murine MC3T3-E1 cells in culture: An in vitro model of osteoblast development. J Bone Miner Res. 1992; 7(6):683-692. doi:10.1002/jbmr.5650070613
17. Kanzler B, Kuschert SJ, Liu YH, Mallo M. Hoxa-2 restricts the chondrogenic domain and inhibits bone formation during development of the branchial area. Development. 1998;125(14):2587-2597. PMID: 9636074

18. Hu R, Liu W, Li H, et al. A Runx2/miR-3960/miR-2861 regulatory feedback loop during mouse osteoblast differentiation. J Biol Chem. 2011; 286(14):12328-12339. doi:10.1074/jbc.M110.176099

19. Xie Q, Wang Z, Zhou H, et al. The role of miR-135-modified adiposederived mesenchymal stem cells in bone regeneration. Biomaterials. 2016;75:279-294. doi:10.1016/j.biomaterials.2015.10.042

20. da Silva RA, Fuhler GM, Janmaat VT, et al. HOXA cluster gene expression during osteoblast differentiation involves epigenetic control. Bone. 2019;125:74-86. doi:10.1016/j.bone.2019.04.026

21. Naylor K, Eastell R. Bone turnover markers: Use in osteoporosis. Nat Rev Rheumatol. 2012;8(7):379-389. doi:10.1038/nrrheum.2012.86

22. Takahashi S. Bone metabolic markers for evaluation of bone metastases [in Japanese]. Clin Calcium. 2013;23:391-400. PMID:23445893

23. Komori T. Regulation of osteoblast differentiation by transcription factors. J Cell Biochem. 2006;99(5):1233-1239. doi:10.1002/jcb.20958

24. Martin TJ. Historically significant events in the discovery of RANK/ RANKL/OPG. World JOrthop. 2013;4(4):186-197. doi:10.5312/wjo.v4.i4.186

25. Martin TJ, Sims NA. RANKL/OPG: Critical role in bone physiology. Rev Endocr Metab Disord. 2015;16(2):131-139. doi:10.1007/s11154-0149308-6

26. Lane NE, Kelman A. A review of anabolic therapies for osteoporosis. Arthritis Res Ther. 2003;5(5):214-222. doi:10.1186/ar797

27. Marx RE, Garg AK. Bone structure, metabolism, and physiology: Its impact on dental implantology. Implant Dent. 1998;7:267-276. doi:10. 1097/00008505-199807040-00004

28. Gersch RP, Lombardo F, McGovern SC, Hadjiargyrou M. Reactivation of Hox gene expression during bone regeneration. J Orthop Res. 2005;23(4):882-890. doi:10.1016/j.orthres.2005.02.005

29. Dobreva G, Chahrour M, Dautzenberg M, et al. SATB2 is a multifunctional determinant of craniofacial patterning and osteoblast differentiation. Cell. 2006;125(5):971-986. doi:10.1016/j.cell.2006.05.012

30. Ellies DL, Krumlauf R. Bone formation: The nuclear matrix reloaded. Cell. 2006;125(5):840-842. doi:10.1016/j.cell.2006.05.022

31. Yang $X$, Jiang $T$, Wang $Y$, Guo $L$. The role and mechanism of SIRT1 in resveratrol-regulated osteoblast autophagy in osteoporosis rats. Sci Rep. 2019;9(1):18424. doi:10.1038/s41598-019-44766-3

32. Liu P, Baumgart M, Groth M, et al. Dicer ablation in osteoblasts by Runx2 driven cre-loxP recombination affects bone integrity, but not glucocorticoid-induced suppression of bone formation. Sci Rep. 2016;6:32112.doi:10.1038/srep32112

33. Shen GY, Ren $\mathrm{H}$, Shang $\mathrm{Q}$, et al. Let-7f-5p regulates TGFBR1 in glucocorticoid-inhibited osteoblast differentiation and ameliorates glucocorticoid-induced bone loss. Int J Biol Sci. 2019;15(10):2182-2197. doi:10.7150/ijbs.33490

34. Bruderer M, Richards RG, Alini M, Stoddart MJ. Role and regulation of RUNX2 in osteogenesis. Eur Cell Mater. 2014;28:269-286. doi:10. 22203/ecm.v028a19

35. Pratap J, Galindo M, Zaidi SK, et al. Cell growth regulatory role of RUNX2 during proliferative expansion of preosteoblasts. Cancer Res. 2003;63(17):5357-5362. PMID:14500368

36. Franceschetti T, Dole NS, Kessler CB, Lee SK, Delany AM. Pathway analysis of microRNA expression profile during murine osteoclastogenesis. PLoS One. 2014;9:e107262. doi:10.1371/journal.pone.0107262

37. Baker N, Sohn J, Tuan RS. Promotion of human mesenchymal stem cell osteogenesis by PI3-kinase/Akt signaling, and the influence of caveolin-1/cholesterol homeostasis. Stem Cell Res Ther. 2015;6:238. doi:10.1186/s13287-015-0225-8

38. Baroncelli M, Fuhler GM, van de Peppel J, et al. Human mesenchymal stromal cells in adhesion to cell-derived extracellular matrix and titanium: Comparative kinome profile analysis. J Cell Physiol. 2019;234(3):2984-2996. doi:10.1002/jcp.27116

39. Chapurlat RD, Confavreux CB. Novel biological markers of bone: From bone metabolism to bone physiology. Rheumatology (Oxford). 2016; 55(10):1714-1722. doi:10.1093/rheumatology/kev410 